\title{
Local Electrical Imaging of Tetragonal Domains and Field-Induced Ferroelectric Twin Walls in Conducting $\mathrm{SrTiO}_{3}$
}

\author{
H. J. Harsan Ma, ${ }^{1,2}$ S. Scharinger, ${ }^{3}$ S. W. Zeng, ${ }^{1,2}$ D. Kohlberger, ${ }^{3}$ M. Lange, ${ }^{3}$ A. Stöhr, ${ }^{3}$ X. Renshaw Wang, ${ }^{1,2}$ \\ T. Venkatesan, ${ }^{1,2,4}$ R. Kleiner, ${ }^{3}$ J. F. Scott, ${ }^{5}$ J. M. D. Coey, ${ }^{1,6}$ D. Koelle, ${ }^{3, *}$ and Ariando ${ }^{1,2, \dagger}$ \\ ${ }^{1}$ NUSNNI-Nanocore, National University of Singapore, 117411 Singapore, Singapore \\ ${ }^{2}$ Department of Physics, National University of Singapore, 117542 Singapore, Singapore \\ ${ }^{3}$ Physikalisches Institut and Center for Quantum Science (CQ) in LISA ${ }^{+}$, Universität Tübingen, Auf der Morgenstelle 14, \\ D-72076 Tübingen, Germany \\ ${ }^{4}$ Department of Electrical and Computer Engineering, National University of Singapore, 117576 Singapore, Singapore \\ ${ }^{5}$ School of Chemistry and School of Physics, St. Andrews University, St. Andrews KY16 9ST, United Kingdom \\ ${ }^{6}$ Department of Pure and Applied Physics, Trinity College, Dublin 2, Ireland \\ (Received 5 May 2015; revised manuscript received 22 March 2016; published 20 June 2016)
}

\begin{abstract}
We demonstrate electrical mapping of tetragonal domains and electric field-induced twin walls in $\mathrm{SrTiO}_{3}$ as a function of temperature and gate bias utilizing the conducting $\mathrm{LaAlO}_{3} / \mathrm{SrTiO}_{3}$ interface and low-temperature scanning electron microscopy. Conducting twin walls appear below $105 \mathrm{~K}$, and new twin patterns are observed after thermal cycling through the transition or on electric field gating. The nature of the twin walls is confirmed by calculating their intersection angles for different substrate orientations. Numerous walls formed when a large side- or back-gate voltage is applied are identified as field-induced ferroelectric twin walls in the paraelectric tetragonal matrix. The walls persist after switching off the electric field and on thermal cycling below $105 \mathrm{~K}$. These observations point to a new type of ferroelectric functionality in $\mathrm{SrTiO}_{3}$, which could be exploited together with magnetism and superconductivity in a multifunctional context.
\end{abstract}

DOI: 10.1103/PhysRevLett.116.257601

The emerging field of domain boundary engineering requires interfaces with unique functionalities [1] such as in the $\mathrm{SrTiO}_{3}$ (STO)-based heterostructures [2-5]. STO is cubic at room temperature, but undergoes a ferroelastic transition to tetragonal structure at around $105 \mathrm{~K}$. It does not become spontaneously ferroelectric at low temperatures despite its huge permittivity [6,7]. Nevertheless, electric order can be induced by stress, or by electric field $(E)$ [8-10] at a threshold of $1.40 \mathrm{kV} / \mathrm{cm}$ at $\sim 5 \mathrm{~K}$.

When STO is used as a substrate or gate insulator for materials such as topological insulators and superconductors $[11,12]$, films grown on STO are assumed to be biased uniformly. However, this is not really the case as nonuniformity can arise from the tetragonal domain structure. Twin boundaries between domains (twin walls) in STO are of particular interest, as they have been suggested to become conducting and ferroelectric at low temperatures [13-19]. Further, it has been demonstrated that ferroelectric "stripes" can be injected in ferroelectric thin films by increasing the applied voltage $[20,21]$, which requires the material, or at least its domain walls, to be ferroelectric. Here, we image the ferroelastic twin walls in STO, and show that their response above the threshold field is strong evidence that it is the field-induced twin walls that become ferroelectric.

STO can be made conducting by doping, by oxygen vacancies, or by electronic reconstruction induced by a polar oxide $\mathrm{LaAlO}_{3}$ (LAO) that produces a few nanometers of two-dimensional electron gas (2DEG) below the interface [22-24], while the bulk of the STO remains insulating. Recently, an influence of the local tetragonal domain structure on the conductivity of the 2DEG at the LAO/ STO interface was demonstrated by probing the magnetic field [25] or electric potential [26] induced by the 2DEG, rather than mapping the electronic properties directly, which is difficult with a probe such as scanning tunneling microscopy because the 2DEG is embedded between two insulating oxides. A method that can map the local electric transport properties right at the interface is low-temperature scanning electron microscopy (LTSEM) [27-31], which uses a periodically blanked focused electron beam ( $e$ beam) scanned across the sample [29-31]. It locally perturbs its electric conductivity with micron-scale spatial resolution and induces a voltage signal $\Delta V$ across the sample. The signal $\Delta V$ gives access to the electronic properties of the 2DEG directly.

In our experiments (see Supplemental Material [32]), the 2DEG microbridges are obtained by growing and patterning a thin LAO film on an STO substrate, with either (001) or (110) orientation. The orientation of the microbridges is described in Table I. LTSEM is utilized to obtain micronscale maps of the $e$-beam-induced $\Delta V$ across the microbridges, imaging the twin walls via an enhanced contrast due to the beam-induced perturbation of the local 
TABLE I. Summary of device characteristics used in this study, listing LAO thicknesses and the (expected and observed) intersection angles of twin walls in the plane of the substrate surface with respect to the long direction of the microbridges. Note that the microbridges of STO(001) samples are along either [100] or [010], which are not distinguished. For the STO(110) samples, "a" represents microbridges along [001] and "b" along [11̄0].

\begin{tabular}{|c|c|c|c|c|c|c|c|}
\hline \multicolumn{4}{|c|}{$\mathrm{STO}(001)$} & \multicolumn{4}{|c|}{ STO(110) } \\
\hline \multirow[b]{2}{*}{ Sample } & \multirow[b]{2}{*}{ LAO thickness (unit cell) } & \multicolumn{2}{|c|}{ Pattern angles (deg) } & \multirow[b]{2}{*}{ Sample } & \multirow[b]{2}{*}{ LAO thickness (unit cell) } & \multicolumn{2}{|c|}{ Pattern angles (deg) } \\
\hline & & Expected & Observed & & & Expected & Observed \\
\hline No. 1 & 5 & $0,90,45,135$ & $0,90,135$ & No. $5 \mathrm{a}$ & 5 & $0,55,125$ & 55,125 \\
\hline No. 2 & 20 & $0,90,45,135$ & 0,90 & No. $5 b$ & 5 & $35,90,145$ & $35,90,145$ \\
\hline No. 3 & 5 & $0,90,45,135$ & $0,90,135$ & No. 6a & 10 & $0,55,125$ & 55,125 \\
\hline No. 4 & 10 & $0,90,45,135$ & $0,90,45$ & No. $6 b$ & 10 & $35,90,145$ & $35,90,145$ \\
\hline
\end{tabular}

conductivity of the 2DEG. The experimental setup is illustrated in Fig. 1, together with a backscattered electron (BSE) surface image and a LTSEM $\Delta V$ map of the STO (001) sample No. 1 at $5 \mathrm{~K}$. Clear stripelike patterns around 1-2 $\mu \mathrm{m}$ wide (the spatial resolution of the instrument) show up in the map, in contrast to the featureless BSE image. Figure 2 shows the evolution of the LTSEM $\Delta V$ maps with increasing temperature for the same sample. The contrast decreases with increasing temperature, and it disappears just above $105 \mathrm{~K}$ [Fig. 2(b)]. The persistent wall signals above $105 \mathrm{~K}$ are attributed to a conducting footprint of oxygen vacancies left after the wall has gone [33]. In Fig. 2(c), which includes the leads, stripes that are along either [100] or [010] are seen, which are associated with twin walls in STO below the transition. The contrast of the (bright) signals on the entire microbridge decreases with increasing temperature, although the total resistance $R$ of the bridge and also its derivative $d R / d T$ increase with $T$, indicating that the voltage signal is not due to beam-induced local heating [27]. The voltage contrast due

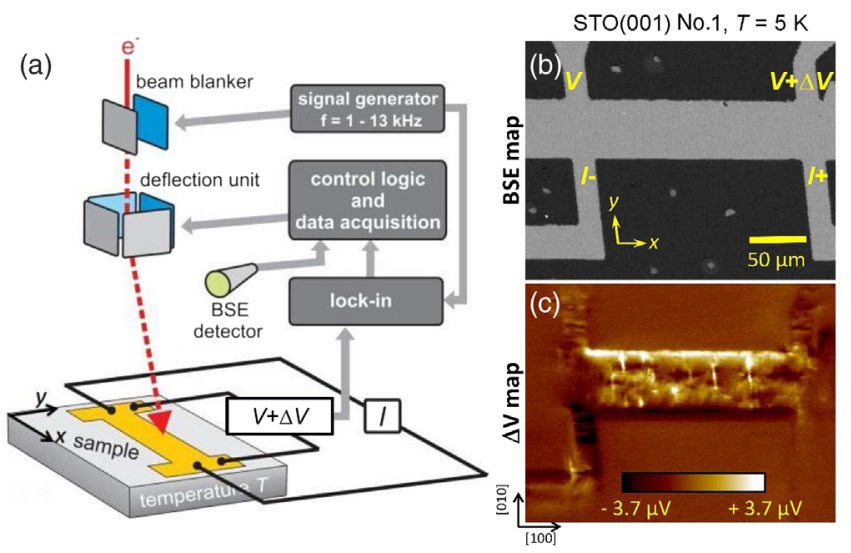

FIG. 1. (a) The measurement setup with an $e$ beam scanning across the surface of the LAO/STO sample. The $e$ beam is chopped by a beam blanker, while $\Delta V$ is detected using a lock-in technique. (b) BSE and (c) LTSEM voltage image, measured at $5 \mathrm{~K}(I=100 \mu \mathrm{A})$ on the $\mathrm{STO}(001)$ sample No. 1. Clear stripes show up in the voltage signal but not in the BSE signal. The image is slightly tilted by an angle $\varphi_{t}$ due to a thermal drift that is corrected in the analysis of the LTSEM maps. to the injected beam current flowing along the microbridge can also be ruled out as the expected signals are 2 orders of magnitude too small and should increase with $R(T)$, contrary to observation. We hence propose that the $e$ beam locally excites charge carriers (electrons and holes). A fraction of those, with density $\Delta n$, diffuses to the 2DEG, where they are separated in the applied bias field $E=$ $V / L \propto I$ (bias current) and contribute to $\Delta V \propto \Delta n I$. The enhanced signals at twin walls can then be due both to their enhanced intrinsic conductivity and to the enhanced generation of charge carriers at twin walls. We cannot separate these two contributions.

A remarkable observation is the complex patterns of twin walls that appear in both (001)- and (110)-oriented STO samples, some of which are shown in Fig. 3. The twins are denoted as $X[100], Y[010]$, and $Z[001]$, based on the $c$ axis direction in the original cubic cell. A twin wall forms when

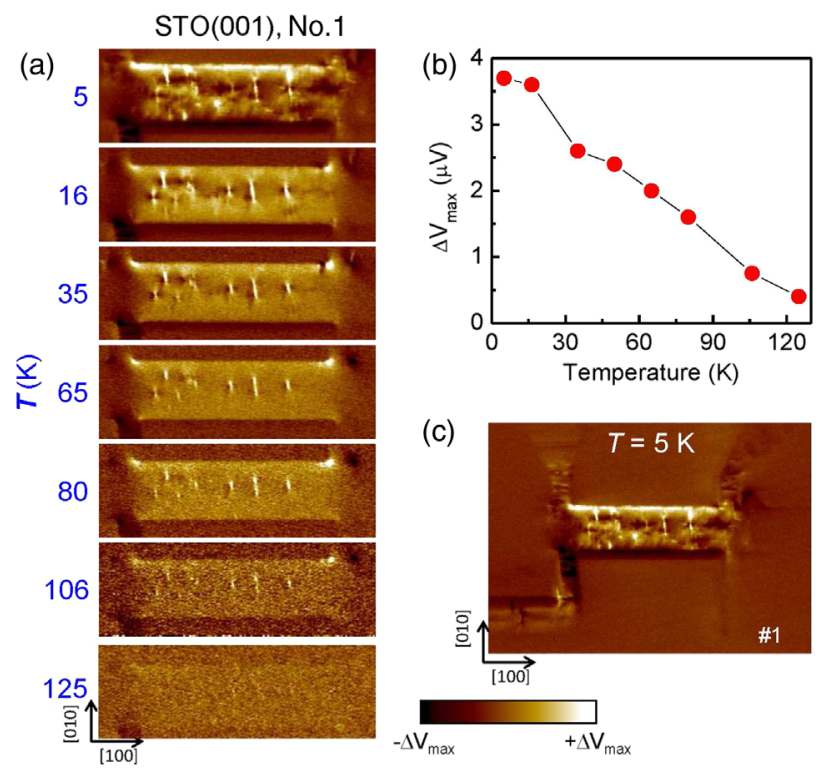

FIG. 2. (a) Series of LTSEM voltage images (with $I=100 \mu \mathrm{A}$ ) at increasing $T$ for sample No. 1 on $\mathrm{STO}(001)$. The $\Delta V$ signal disappears just above $105 \mathrm{~K}$, and the maximum intensity $\Delta V_{\max }$ of the bright stripes is plotted versus temperature in (b). (c) Voltage image observed at $5 \mathrm{~K}$, including the electrodes. 
(a) $\mathrm{STO}(001), T=5 \mathrm{~K}$

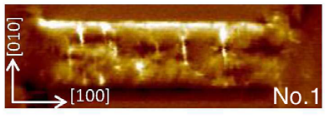

(b)

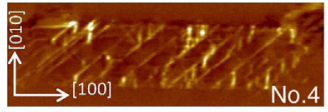

(c) $\mathrm{STO}(110), T=5 \mathrm{~K}$

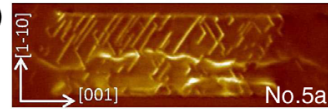

(d)

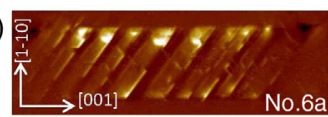

(e)

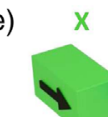

(f)

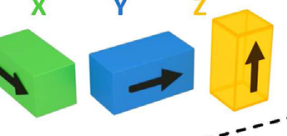

(g)

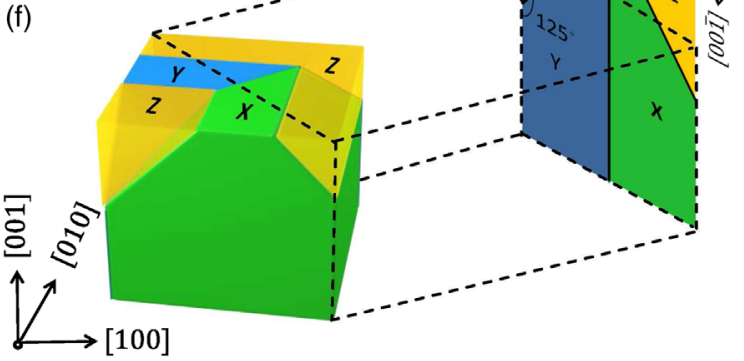

FIG. 3. LTSEM maps of different samples showing different patterns of twin walls; (a) and (b) are for LAO/STO(001), and (c) and (d) are for LAO/STO(110). (a) $0^{\circ}$ (along [100]) and $90^{\circ}$ (along [010]) patterns in sample No. 1. (b) $0^{\circ}$ (along [100]) and $45^{\circ}$ (along [110]) patterns in sample No. 4. (c) $55^{\circ}$ and $125^{\circ}$ patterns in sample No. 5 a. (d) $55^{\circ}$ and $125^{\circ}$ patterns in sample No. 6a. (e) Three kinds of tetragonal domains in STO, $X, Y$, and $Z$, have their $c$ axis along [100], [010], and [001], respectively. (f) A three-dimensional schematic of different twins and their walls in a cubic coordinate system. (g) Cross section of twins in the (110) plane. The angle of the twin wall between $Z$ and $X(Y)$ is either $55^{\circ}\left(125^{\circ}\right)$ or $145^{\circ}\left(35^{\circ}\right)$ in the $(110)$ plane. The twin wall between $X$ and $Y$ intersects along [001] in the (110) plane.

adjacent domains share an $a$ axis [26]. Although the $c$ axes are always orthogonal, the twin boundaries can intersect at different angles in the (001) or (110) plane of the STO surface. The calculated angles and those observed in our various samples are listed in Table I. In Figs. 3(a) and 3(b), the twin walls are along [100], [010], or [110], which correspond to angles of $0^{\circ}, 90^{\circ}$, or $45^{\circ}$, respectively, in the (001) plane. In Figs. 3(c) and 3(d), the twin walls make angles close to $55^{\circ}, 125^{\circ}$, or $145^{\circ}$ [see Table I and Figs. 3(f) and $3(\mathrm{~g})]$. In the (001) plane, $X$ and $Y$ form twin walls that intersect at $45^{\circ}$ to [010]; $Y$ and $Z$ intersect at $90^{\circ} ; Z$ and $X$ intersect at $0^{\circ}$, as shown in Fig. 3(f). In order to explain the angles observed in the (110) plane, we calculated the intersection between the twin wall and the substrate orientation. In Fig. 3(g), we show that the angle of the twin wall between $Z$ and $X(Y)$ is either $55^{\circ}\left(125^{\circ}\right)$ or $145^{\circ}$ $\left(35^{\circ}\right)$. The twin wall between $X$ and $Y$ has an intersection along [001]. These are exactly what we observe and we conclude that we are imaging the twin boundaries between different tetragonal domains. Our results are consistent with those in Ref. $[25,26,34]$ and with recent results on optical polarization microscopy [35] (also see Supplemental
Material [32]). Moreover, after thermal cycling to above $125 \mathrm{~K}$, the spatial configuration of the stripe patterns in $\Delta V$ images clearly changes, which supports our claim that we are indeed imaging twin walls.

To investigate the response of the twin walls to electric field, we apply $E$-fields to the samples using a side [Fig. 4(a)] or back gate [Fig. 4(b)]. The distance to the side-gate electrode is $165 \mu \mathrm{m}$, and that to the back gate electrode is $500 \mu \mathrm{m}$. The threshold of about $1.4 \mathrm{kV} / \mathrm{cm}$ for the appearance of ferroelectricity in STO [9] therefore corresponds to a side-gate voltage of $23 \mathrm{~V}$ or a back-gate voltage of $70 \mathrm{~V}$. Figure 4(c) shows a sequence of LTSEM maps at different side-gate voltages from 0 to $-200 \mathrm{~V}$ at $5 \mathrm{~K}$. With a $-200 \mathrm{~V}$ gate field, many narrow twin walls are created, and they remain after switching off the gate voltage, and even after thermal cycling to $80-100 \mathrm{~K}$ for 12 hours. Most of them disappear after thermal cycling to $300 \mathrm{~K}$. Figure 4(d) shows LTSEM voltage maps at different back-gate voltage from 0 to $-210 \mathrm{~V}$ at $5 \mathrm{~K}$. The results are similar to those for the side gate. We also show that

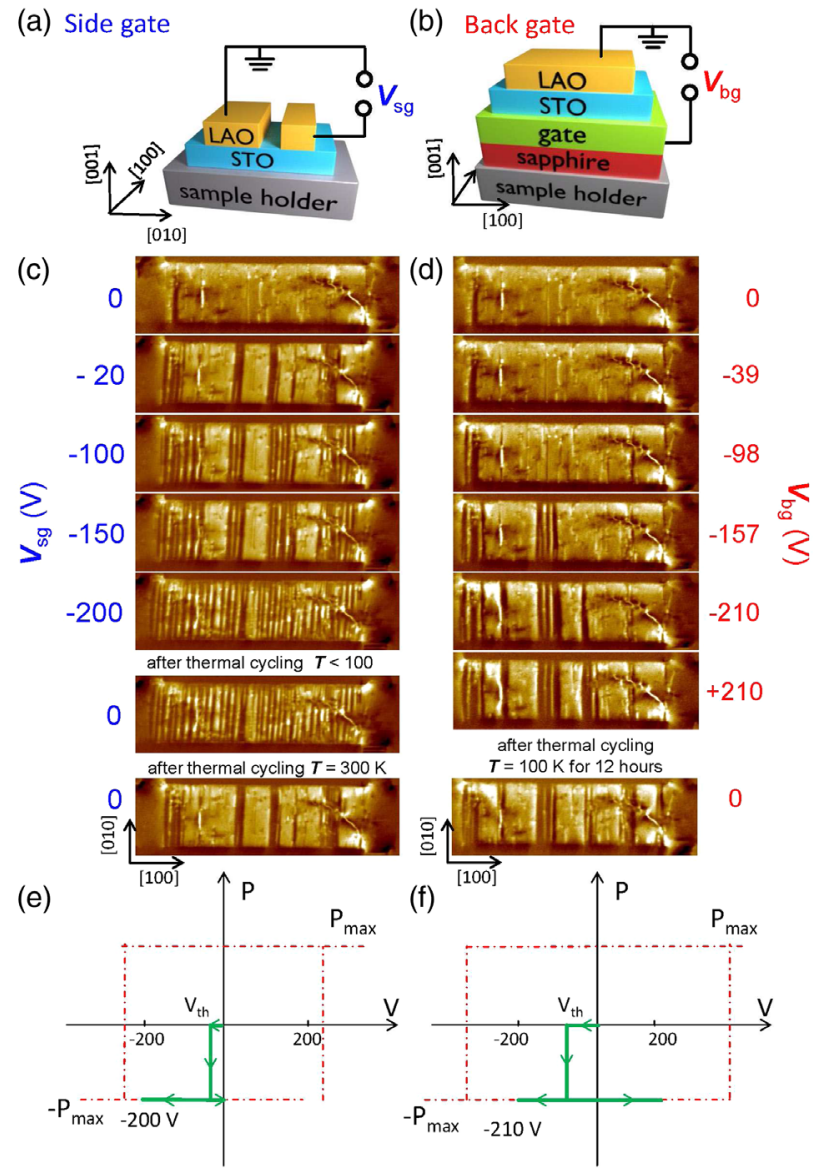

FIG. 4. Schematics showing (a) the side- and (b) back-gate configurations. (c) LTSEM $\Delta V$ images for different side-gate voltages from 0 to $-200 \mathrm{~V}$ at $5 \mathrm{~K}$ for (001) sample No. 1. (d) LTSEM $\Delta V$ images for different back-gate voltages from 0 to -210 V. (e,f) Schematics of electric field effect on the ferroelectric state of the STO in the side- (e) and back-gate (f) case. 
applying a positive back-gate voltage does not erase the twin walls. The number of twin walls induced with the $E$-field is plotted in Fig. 5 for both side- (blue square) and back-gate (red circles) configurations. The intercept at $1.4-1.5 \mathrm{kV} / \mathrm{cm}$ corresponds to the onset of field-induced electric order in STO at $5 \mathrm{~K}$, indicating that the $E$-fieldinduced twin walls above the threshold are ferroelectric. We do not, however, observe a ferroelectric hysteresis loop when we reverse the gate voltage from -200 to $200 \mathrm{~V}$ for either the side or back gate, possibly because the maximum applied fields $(\sim 10 \mathrm{kV} / \mathrm{cm})$ are rather small and do not allow a full hysteresis loop scan [Figs. 4(e) and 4(f)]. As $\Delta V$ [Fig. 2(b)] is monitored at constant current, the brighter regions correspond to areas where either the conductance or the beam-induced change of conductance is higher than average. This we think occurs in the vicinity of conducting twin walls [Figs. 1(c), 2(a) and 2(c), and 3(a)-3(d)] $[17,18,25]$. However, when the walls become ferroelectrically polarized, the situation is different, as shown in Figs. 4(c) and 4(d). On either side of the wall there is a dark band that seems to be insulating, like the surrounding background outside the microbridge. It appears that the 2DEG is depleted for some distance when the wall becomes ferroelectric, forming a Schottky-like barrier.

Normally different segments of a polar wall may have dipole moments pointing in different directions within the plane, some along the direction of the apices of the $\mathrm{TiO}_{6}$ octahedra in the twin wall, and others in the opposite direction forming polarizations, which can be stabilized by an in-plane $E$-field [15]. The polarity of the domain walls is enhanced by applying an $E$-field parallel to the plane of the twin wall, which aligns the polar clusters within the twin wall and induces ferroelectricity. It was reported that the low-temperature heat capacity of STO under an $E$-field has an additional contribution from excitations of unknown origin that might be related to field-induced polarization and ferroelectricity [9]. We interpret the bright lines in the images of Fig. 4(c) and 4(d) as the twin walls, while the dark yellow stripes are the domains between walls. Our results suggest that the field-induced ferroelectricity in

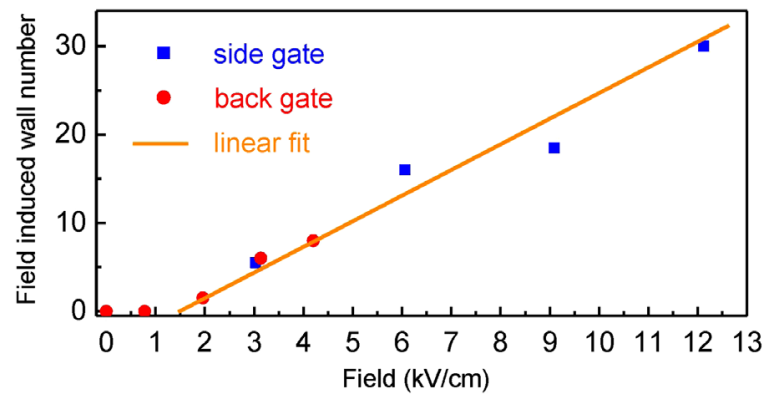

FIG. 5. The back- and side-gate field-induced wall numbers versus the applied gate field. The fitted (dashed) line intersects with the $E$-field axis at around $1.5 \mathrm{kV} / \mathrm{cm}$, which is the onset of the $E$-field-induced electric ordering in STO at $5 \mathrm{~K}$.
STO occurs only within the walls, since the size of the domains between twin walls does not increase when we increase the gate voltage.

The twin walls do not entirely disappear on heating above $300 \mathrm{~K}$ and cooling back again to $5 \mathrm{~K}$ [Figs. 4(c) and 4(d)]. One would expect that on bringing the STO in the cubic phase all twin walls should disappear, but the material seems to surprisingly "remember" its twin-wall state, which resembles very much that of the $-20 \mathrm{~V}$ case in the sidegate case after thermal cycling at $300 \mathrm{~K}$. Since the walls are conducting we think they become electrically charged during the long exposure to voltage bias at $5 \mathrm{~K}$ $[17,18,25]$. This charge is pinned and creates charged sections at the position of the twin walls, then shows up in similar positions on going below $105 \mathrm{~K}$ again. This memory effect might be tied to inevitable defects in the samples. When temperature decreases from above the transition to below, the twin walls start to form at the defects and then expand to other areas with decreasing temperature. Since defects are now present, they can be more easily charged by a bias current or electric field. Another possible reason for the memory effect of ferroelastic or ferroelectric twin walls is the persistence of a residual electric field in STO after applying a large electric field [8].

The reason for the appearance of multiple ferroelectric twin walls rather than a bulk ferroelectric state may be understood by considering the depolarizing field $E_{d}$,

$$
\epsilon_{0} E_{d}=\langle P\rangle=-P \text { tn },
$$

where $\langle P\rangle$ is the average polarization, $P$ is the electric polarization of a twin wall, $t$ is the wall thickness, and $n$ is the number of walls per meter. When the polarization is stable, the net field, $E+E_{d}$, is 0 . The slope of Fig. 5 gives $E / n=-E_{d} / n \sim 6.0 \mathrm{~V}$. Hence, $P t=6.0 \times \epsilon_{0}$. Assuming an effective wall thickness of $1 \mathrm{~nm}$, we deduce a polarization of $0.05 \mathrm{C} \mathrm{m}^{-2}$, which is a reasonable value for a ferroelectric film. One can expect to produce twin walls by applying an $E$-field to a material with polar ferroelastic walls that need to balance the depolarization field created by the charges associated with the ferroelectric sheet $[36,37]$. They may also become electrically conducting due to the charge injected into them from the gate [17]. Though a transition to an orthorhombic ferroelectric phase of STO has been deduced from analysis of neutron diffraction superlattice reflections under applied field or strain [8], to the best of our knowledge, this is the first observation in real space of $E$-field-induced ferroelectric twin walls in a nonferroelectric bulk material.

Coupling of different order parameters opens the door for a multitude of novel effects on the nanometer length scale of the thickness of a twin wall [38]. Besides the ferroelasticity below $105 \mathrm{~K}$ and field-induced electric ordering at low temperature, STO has been shown to be superconducting $[4,5]$ below $300 \mathrm{mK}$ and ferromagnetic even at room temperature $[3,39]$. The conducting twin 
walls could play the role of electric wiring in multiferroic domain wall devices. In future work, possible coupling among these intriguing properties will have to be explored.

In conclusion, LTSEM has enabled us to image the tetragonal twin walls in STO below $105 \mathrm{~K}$ by mapping the local electric transport properties of the interfacial 2DEG. Although the technique has only micron-scale spatial resolution, it is sensitive to the presence of twin walls and planar ferroelectric defects that are only about a nanometer thick. We find that the $E$-field, beyond a critical threshold, induces ferroelectricity within the twin walls in STO, and creates an increasing density of them with increasing field. These observations are relevant for the low-temperature properties of a wide variety of complex oxide heterostructures that are routinely built on STO and are therefore sensitive to its structure and phase transitions. Our work demonstrates the prospect of designing electronic systems at the nanoscale (the thickness of a twin wall) by making use of the ferroelectric twin walls in conducting STO-based perovskite heterostructures.

We gratefully acknowledge helpful discussions with Yiftach Frenkel. This work is supported by the National University of Singapore (NUS) Academic Research Fund (AcRF Tier 1 Grants No. R-144-000-346-112 and No. R144-000-364-112) and the Singapore National Research Foundation (NRF) under the Competitive Research Programs (CRP Awards No. NRF-CRP 8-2011-06 and No. NRF-CRP10-2012-02) by the Institutional Strategy of the University of Tübingen (Deutsche Forschungsgemeinschaft, Grant No. ZUK 63), and by the EU-FP6-COST Grant No. MP1308.

*koelle@uni-tuebingen.de †ariando@nus.edu.sg

[1] E. K. H. Salje, Phase Transitions 86, 2 (2013).

[2] A. Brinkman, M. Huijben, M. van Zalk, J. Huijben, U. Zeitler, J. C. Maan, W. G. van der Wiel, G. Rijnders, D. H. A. Blank, and H. Hilgenkamp, Nat. Mater. 6, 493 (2007).

[3] Ariando et al., Nat. Commun. 2, 188 (2011).

[4] J. F. Schooley, W. R. Hosler, and M. L. Cohen, Phys. Rev. Lett. 12, 474 (1964).

[5] N. Reyren et al., Science 317, 1196 (2007).

[6] K. A. Müller and H. Burkard, Phys. Rev. B 19, 3593 (1979).

[7] R. C. Neville, B. Hoeneisen, and C. A. Mead, J. Appl. Phys. 43, 2124 (1972).

[8] J. Sidoruk, J. Leist, H. Gibhardt, M. Meven, K. Hradil, and G. Eckold, J. Phys. Condens. Matter 22, 235903 (2010).

[9] J. Hemberger, M. Nicklas, R. Viana, P. Lunkenheimer, A. Loidl, and R. Böhmer, J. Phys. Condens. Matter 8, 4673 (1996).

[10] E. Hegenbarth, Phys. Status Solidi 6, 333 (1964).

[11] C.-Z. Chang et al., Science 340, 167 (2013).

[12] K. A. Parendo, K. H. S. B. Tan, A. Bhattacharya, M. EblenZayas, N. E. Staley, and A. M. Goldman, Phys. Rev. Lett. 94, 197004 (2005).
[13] J. F. Scott, E. K. H. Salje, and M. A. Carpenter, Phys. Rev. Lett. 109, 187601 (2012).

[14] E. K. H. Salje, O. Aktas, M. A. Carpenter, V. V. Laguta, and J. F. Scott, Phys. Rev. Lett. 111, 247603 (2013).

[15] T. Zykova-Timan and E. K. H. Salje, Appl. Phys. Lett. 104, 082907 (2014).

[16] P. A. Fleury, J. F. Scott, and J. M. Worlock, Phys. Rev. Lett. 21, 16 (1968).

[17] J. Seidel et al., Nat. Mater. 8, 229 (2009).

[18] J. Guyonnet, I. Gaponenko, S. Gariglio, and P. Paruch, Adv. Mater. 23, 5377 (2011).

[19] S. Farokhipoor and B. Noheda, J. Appl. Phys. 112, 052003 (2012).

[20] J. Lajzerowicz and J. J. Niez, J. Phys. (Paris) Lett. 40, 165 (1979).

[21] J. R. Whyte, R. G. P. McQuaid, P. Sharma, C. Canalias, J. F. Scott, A. Gruverman, and J. M. Gregg, Adv. Mater. 26, 293 (2014).

[22] A. Ohtomo and H. Y. Hwang, Nature (London) 427, 423 (2004).

[23] H. J. H. Ma, Z. Huang, W. M. Lü, A. Annadi, S. W. Zeng, L. M. Wong, S. J. Wang, T. Venkatesan, and Ariando, Appl. Phys. Lett. 105, 011603 (2014).

[24] M. Basletic, J.-L. Maurice, C. Carrétéro, G. Herranz, O. Copie, M. Bibes, É. Jacquet, K. Bouzehouane, S. Fusil, and A. Barthélémy, Nat. Mater. 7, 621 (2008).

[25] B. Kalisky et al., Nat. Mater. 12, 1091 (2013).

[26] M. Honig, J. A. Sulpizio, J. Drori, A. Joshua, E. Zeldov, and S. Ilani, Nat. Mater. 12, 1112 (2013).

[27] R. Gross and D. Koelle, Rep. Prog. Phys. 57, 651 (1994).

[28] S. Guénon, S. Scharinger, S. Wang, J. G. Ramírez, D. Koelle, R. Kleiner, and I. K. Schuller, Europhys. Lett. 101, 57003 (2013).

[29] D. Doenitz, R. Straub, R. Kleiner, and D. Koelle, Appl. Phys. Lett. 85, 5938 (2004).

[30] C. Gürlich, S. Scharinger, M. Weides, H. Kohlstedt, R. G. Mints, E. Goldobin, D. Koelle, and R. Kleiner, Phys. Rev. B 81, 094502 (2010).

[31] C. Gürlich, E. Goldobin, R. Straub, D. Doenitz, Ariando, H.-J. H. Smilde, H. Hilgenkamp, R. Kleiner, and D. Koelle, Phys. Rev. Lett. 103, 067011 (2009).

[32] See Supplemental Material at http://link.aps.org/ supplemental/10.1103/PhysRevLett.116.257601 for sample fabrication, experimental techniques, thermal cycling experiments and optical imaging of twin walls.

[33] I. Stolichnov, M. Iwanowska, E. Colla, B. Ziegler, I. Gaponenko, P. Paruch, M. Huijben, G. Rijnders, and N. Setter, Appl. Phys. Lett. 104, 132902 (2014).

[34] A. Buckley, J. P. Rivera, and E. K. H. Salje, J. Appl. Phys. 86, 1653 (1999).

[35] Z. Erlich et al., J. Supercond. Novel Magn. 28, 1017 (2015).

[36] G. Catalan, J. F. Scott, A. Schilling, and J. M. Gregg, J. Phys. Condens. Matter 19, 022201 (2007).

[37] J. F. Scott, J. Phys. Condens. Matter 26, 212202 (2014).

[38] E. K. H. Salje and J. C. Lashley, Domain Boundary Engineering in Ferroic and Multiferroic Materials: A Simple Introduction Springer Series in Materials Science, (Springer, Berlin, Heidelberg, 2012) Vol. 148, pp. 1-18.

[39] F. Bi, M. Huang, S. Ryu, H. Lee, C.-W. Bark, C.-B. Eom, P. Irvin, and J. Levy, Nat. Commun. 5, 5019 (2014). 